\title{
Generalized Analysis of a Distributed Energy Efficient Algorithm for Change Detection
}

\author{
Taposh Banerjee \\ Dept. of ECE, Indian Institute of Science \\ Bangalore, India \\ taposh@gmail.com
}

\author{
Vinod Sharma \\ Dept. of ECE, Indian Institute of Science \\ Bangalore, India \\ vinod@ece.iisc.ernet.in
}

\begin{abstract}
An energy efficient distributed Change Detection scheme based on Page's CUSUM algorithm was presented in [2]. In this paper we consider a nonparametric version of this algorithm. In the algorithm in [2], each sensor runs CUSUM and transmits only when the CUSUM is above some threshold. The transmissions from the sensors are fused at the physical layer. The channel is modeled as a Multiple Access Channel (MAC) corrupted with noise. The fusion center performs another CUSUM to detect the change. In this paper, we generalize the algorithm to also include nonparametric CUSUM and provide a unified analysis.
\end{abstract}

\section{Categories and Subject Descriptors}

G.3 [Probability and Statistics]: distributed functions, renewal theory, queueing theory, stochastic processes; H.3.4 [Systems and Software]: distributed systems

\section{General Terms}

Algorithms, Design, Performance, Theory

\section{Keywords}

Nonparametric CUSUM, Decentralized Change Detection, Reflected Random Walk.

\section{INTRODUCTION}

In the problem of distributed change detection, there are multiple geographically distributed sensors, each sensing a sequence of observations. The distribution of the observations of all the sensors changes simultaneously at some random point of time, and the observations are independent and identically distributed (iid) conditioned on the time of change. The sensors send processed version (e.g., scaled or quantized) of their observations to a decision maker (fusion center) and the fusion center fuses the information from various sensors to detect the change of law as soon as possible. The performance metric is a measure of the number of extra observations taken after the distribution has changed under some false alarm constraint (the constraint corresponds to the cost associated with declaring a change when it has not taken place). This

Permission to make digital or hard copies of all or part of this work for personal or classroom use is granted without fee provided that copies are not made or distributed for profit or commercial advantage and that copies bear this notice and the full citation on the first page. To copy otherwise, to republish, to post on servers or to redistribute to lists, requires prior specific permission and/or a fee.

MSWiM'09, October 26-29, 2009, Tenerife, Canary Islands, Spain

Copyright 2009 ACM 978-1-60558-616-9/09/10 ...\$10.00. model finds application in biomedical signal processing, intrusion detection in computer and sensor networks ([19], [18]), finance, quality control engineering, and recently, distributed detection of the primary in cognitive radio networks ([7], [15]).

In the Bayesian formulation of the change detection problem, where the distribution of the change variable is known, the objective is to minimize the mean delay of detection subject to probability of false alarm. The optimal solution is obtained in [16] when the sensors transmit the raw observations (and hence a centralized solution is feasible), and in [19] when the sensors transmit a quantized version of the observations.

In the Min-Max formulation, when no knowledge is assumed of the distribution of change, the worst case delay is minimized subject to a constraint on the mean time to false alarm. When the sensors send the observations in raw, the CUSUM algorithm (first proposed by Page in [12]) was shown to be optimal in [8] and [11]. When the sensors process the information before transmitting, CUSUM based schemes were shown to be optimal in [10].

The above algorithms minimize delay subject to constraints on only false alarm. Hence, it is not clear if they are also energy efficient. As a result, the distributed algorithm which optimizes delay under any of the above two formulations, under both false alarm and energy constraints, is not yet known. Recently, a Bayesian formulation of the decentralized change detection problem with energy constraints was considered in [20]. The problem is solved by restricting the solution to a class of algorithms where the sensors send scaled and shifted versions of their observations to the fusion center. The algorithm in [20] is energy efficient but leaves out a class of algorithms where the sensors can decide not to transmit based on the available information. This latter technique can be used to save more energy.

This issue is exploited in [2] to propose a CUSUM based algorithm called DualCUSUM. This new algorithm was shown to perform much better than the one in [20] and the gap in performance was shown to increase at lower energy and false alarm constraints.

In this paper we provide the false alarm and delay analysis of DualCUSUM. We also extend DualCUSUM to detect changes when the exact information about the pre-change and post-change distributions is not available (nonparametric setting) and provide a unified analysis.

As will be seen from the paper, the analysis of such distributed cooperative systems is very challenging. We use results from Renewal Theory, Extreme Value Theory and theory of Brownian Motion to analyse our algorithm. Since CUSUM is, or will be a fundamental element of many distributed algorithms for detection of change, the tools and techniques used here can be used to analyse those other algorithms as well.

DualCUSUM has been used for cooperative spectrum sensing in 
Cognitive Radio Systems also in [15] and shown to provide better performance than other algorithms available in literature not only in delay in detection but also in saving energy.

The knowledge of pre-change and post-change distributions may not be practical in many cases: random time varying fading in the wireless channels ([5], [7]) and due to lack of information related to transmit power and/or receiver noise ([17]). Thus in this paper we also extend DualCUSUM to a nonparametric set up.

We analyse the generalized version of DualCUSUM of which parametric and nonparametric versions are special cases. A few interesting facts emerge from this analysis: mean detection delay is independent of the distribution of the observations but the false alarm probability crucially depends on the tail behavior of the distributions (at least for the nonparametric case). The lighter the tail, the lower the false alarm probability. Therefore for a given false alarm constraint, under both parametric and nonparametric settings, a system where the observations are lighter tailed, will have lower detection delay.

We also show that the log likelihood function converts a heavy tailed distribution to a light tail distribution, or in general, makes the tail of the distribution lighter. Since, parametric CUSUM uses log likelihood and nonparametric CUSUM does not, the former performs better than the latter for a given distribution of observations. This interesting property of log likelihood and its implications for CUSUM seem to have gone unnoticed so far.

The paper is organized as follows. We explain the model and introduce the algorithm in Section 2 Section 3 analyzes the performance of the algorithm and provides comparison with simulations. Section 4 concludes the paper.

\section{MODEL AND ALGORITHM}

Let there be $L$ sensors in a sensor field, sensing observations and transmitting to a fusion node. The transmissions from the sensor nodes to the fusion node are via a MAC. In our system we assume that all the sensor nodes can transmit at the same time. There is physical layer fusion at the fusion node (commonly studied Gaussian MAC is a special case). The fusion node receives data over time and decides if there is a change in distribution of the observations at the sensors.

Let $X_{k, l}$ be the observation made at sensor $l$ at time $k$. Sensor $l$ transmits $Y_{k, l}$ at time $k$ after processing $X_{k, l}$ and its past observations. The fusion node receives $Y_{k}=\sum_{l=1}^{L} Y_{k, l}+Z_{M A C, k}$, where $\left\{Z_{M A C, k}\right\}$ is iid receiver noise. The distribution of the observations at each sensor changes simultaneously at a random time $T$, with a known distribution. The $\left\{X_{k, l}, l \geq 1\right\}$ are independent and identically distributed (iid) over $l$ and are independent over $k$, conditioned on change time $T$. Before the change $X_{k, l}$ have density $f_{0}$ and after the change the density is $f_{1}$. The expectation under $f_{i}$ will be denoted by $E_{i}, i=0,1$, and $P_{\infty}$ and $P_{1}$ denote the probability measure under no change and when change happens at time 1, respectively. These assumptions are commonly made in the literature (see e.g., [10] and [19])

The objective of the fusion center is to detect this change as soon as possible at time $\tau$ (say) using the messages transmitted from all the $L$ sensors, subject to an upper bound $\alpha$ on the probability of False Alarm $P_{F A} \triangleq P(\tau<T)$ and $\mathscr{E}_{0}$ on the average energy used. Often the desired $\alpha$ is quite low, e.g., $\leq 10^{-6}$ in intrusion detection in sensor networks. Then, the general problem is:

$$
\begin{gathered}
\min E_{D D} \triangleq E\left[(\tau-T)^{+}\right], \\
\text {Subj to } P_{F A} \leq \alpha \text { and } \mathscr{E}_{a v g}=E\left[\sum_{k=1}^{\tau} Y_{k, l}^{2}\right] \leq \mathscr{E}_{0}, 1 \leq l \leq L .
\end{gathered}
$$

Our algorithm in [2] does not provide an optimal solution to the problem but uses several desirable features to provide better performance than the algorithms we are aware of. We reproduce the algorithm, DualCUSUM, for an easy reference:

\section{Sensor $l$ uses CUSUM,}

$$
W_{k, l}=\max \left(0, W_{k-1, l}+\log \left[f_{1}\left(X_{k, l}\right) / f_{0}\left(X_{k, l}\right)\right]\right),
$$$$
\text { where, } W_{0, l}=0,1 \leq l \leq L \text {. }
$$

2. Sensor $l$ transmits $Y_{k, l}=b 1_{\left\{W_{k, l}>\gamma\right\}}$. Here $1_{A}$ denotes the indicator function of set $\mathrm{A}$ and $b$ is a design parameter.

3. Physical layer fusion (as in [20]) is used to reduce transmission delay, i.e., $Y_{k}=\sum_{l=1}^{L} Y_{k, l}+Z_{M A C, k}$, where $Z_{M A C, k}$ is the receiver noise.

4. Finally, Fusion center runs CUSUM:

$$
F_{k}=\max \left\{0, F_{k-1}+\log \frac{g_{I}\left(Y_{k}\right)}{g_{0}\left(Y_{k}\right)}\right\} ; F_{0}=0,
$$

where $g_{0}$ is the density of $Z_{M A C, k}$, the MAC noise at the fusion node, and $g_{I}$ is the density of $Z_{M A C, k}+b I, I$ being a design parameter.

5. The fusion center declares a change at time $\tau(\beta, \gamma, b, I)$ when $F_{k}$ crosses a threshold $\beta: \tau(\beta, \gamma, b, I)=\inf \left\{k: F_{k}>\beta\right\}$.

Multiple values of $(\beta, \gamma, b, I)$ will satisfy both the false alarm and the energy constraint. One can minimize $E_{D D} \triangleq E\left[(\tau-T)^{+}\right]$over this parameter set. In Section 3 we obtain the performance of DualCUSUM for given values $(\beta, \gamma, b, I)$ which then can be used in the optimization algorithm developed in [2] to efficiently solve the optimization problem above.

DualCUSUM, as the original CUSUM itself, has a strong limitation. It requires exact knowledge of $f_{0}$ and $f_{1}$. This information will be available apriori to varying degrees in a practical scenario. Depending upon the type of uncertainty in $f_{0}, f_{1}$, different algorithms/variations on CUSUM are available ([4], [6]). One common algorithm, called nonparametric CUSUM is to replace (2) by

$$
W_{k+1, l}=\max \left\{0, W_{k, l}+X_{k, l}-D\right\},
$$

where, $D$ is an appropriate constant such that $E\left[X_{k, l}-D\right]$ is negative before change and positive after change. If the mean of $X_{k, l}$ is known before and after the change, $D$ can be chosen as the average of the two means. Depending on the uncertainties involved, we should be able to obtain such a $D$. More generally, we should be able to estimate $D$. For Gaussian and exponential distributions, nonparametric CUSUM becomes CUSUM for some appropriate $D$ and scaling. If at the fusion node $g_{0}$ is not known (in our CUSUM algorithm (3) at the fusion node, $g_{I}(x)=I b+g_{0}(x)$ ), then one can use (4) even at the fusion node.

In the following we will compute $P_{F A}$ and $E_{D D}$ for a generalized class of algorithms where at the sensor nodes and at the fusion node we use the algorithm,

$$
W_{k+1}=\max \left\{0, W_{k}+Z_{k+1}\right\},
$$

where, $\left\{Z_{k}\right\}$ is an iid sequence with different distributions before and after the change. We will assume that $E\left[Z_{k}\right]<0$ before the change and $E\left[Z_{k}\right]>0$ after the change. We will denote by $f_{Z}, F_{Z}$ and $P_{Z}$ the density, cdf and probability measure for $Z_{k}$.

Algorithm [5] contains CUSUM and nonparametric CUSUM as special cases. In the next section we analyze the generalized DualCUSUM with (5). We emphasize that unlike DualCUSUM, this algorithm does not require knowledge of $f_{0}$ and $f_{1}$; we only need to chose $D$ appropriately. But, the performance of this algorithm, 
as we show in the next section, does depend on the underlying distributions. This is typical of such algorithms.

\section{ANALYSIS}

In this section, we first compute the false alarm probability $P_{F A}$ and then the delay $E_{D D}$. The idea is to model the times at which the CUSUM $\left\{W_{k}\right\}$ at the local sensors, crosses the threshold $\gamma$ (we drop subscript $l$ for convenience) and the local nodes transmit to the fusion node (Fig. 11).

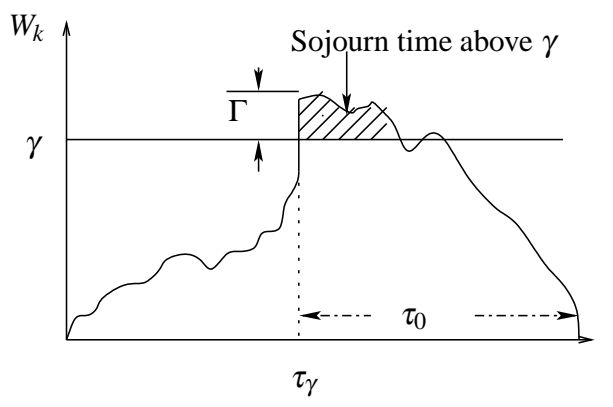

Figure 1: Excursions of $W_{k}$ above $\gamma$ can be approximated by a compound Poisson process. A local node transmits to the fusion node during these excursions.

Computing $P_{F A}$ requires finding (when $Z_{k}$ has distribution $f_{0}$ ) the distribution of $\tau_{\gamma}$, the first time $W_{k}$ crosses $\gamma$, the amount of time it stays above $\gamma$ (sojourn time above $\gamma$ ), and the probability that the fusion node declares a change during a sojourn time. These are computed in Sections 3.1.3.5. Computation of $E_{D D}$ is sketched in Section 3.7

We will need the following notations and definitions. Let $X$ be a random variable with distribution $F$. Then $F^{* n}$ denotes the $n$-fold convolution of $F$ and $\bar{F}(x)=1-F(x)$.

DEFINITION 1. ([1]) $F$ is heavy tailed iffor any $\varepsilon>0, E\left[e^{\varepsilon|X|}\right]=$ $\infty$. $F$ is subexponential if $\bar{F}^{* 2}(x) / \bar{F}(x) \rightarrow 2$ as $x \rightarrow \infty$. If $F$ is not heavy tailed, we call it light tailed.

Gaussian, Exponential and Laplace distributions are light tailed. Pareto, Lognormal and Weibull distributions are subexponential. Subexponential distributions are a subclass of heavy tailed distributions.

Often it is said that light tailed distributions may provide better system behavior than the heavy tailed ([3]). We demonstrate this for the probability of false alarm. In particular we will show that if $F_{Z}$ is light tailed before change then $P_{F A}$ is much less than if it is heavy tailed. Interestingly, we will also show that $E_{D D}$ is largely insensitive to the tail behavior of $F_{Z}$.

CUSUM has the interesting property that it transforms a large class of heavy tailed distributions into light tailed distributions. Consider the class of Pareto distributions:

$$
K \frac{x_{m}^{K}}{x^{(K+1)}} \text { for } x \geq x_{m}, \text { where } x_{m}>0 .
$$

For $f_{0}$, we take $K=(\alpha+1)$ and for $f_{1}, K=\alpha$ where $\alpha$ is a positive constant. Then, if $Z=\log \left(f_{1}(X) / f_{0}(X)\right), P_{\infty}\left[Z_{k}>z\right] \sim e^{-z \alpha}$. On the other hand, light tailed distributions stay light tailed. This important property of log likelihood seems to have escaped the attention of investigators before. This makes CUSUM perform better than the nonparametric CUSUM because as mentioned above, for CUSUM with Pareto distribution, the $P_{F A}$ will be as for a light tailed distribution. But for nonparametric CUSUM, it will be much larger, while the $E_{D D}$ in the two cases will be comparable.

\subsection{Behavior of $W_{k}$ under $P_{\infty}$}

The process $\left\{W_{k}\right\}$ is a reflected random walk with negative drift under $P_{\infty}$. Figure (1) shows a typical sample path for $\left\{W_{k}\right\}$. The process visits 0 (regenerates) a finite number of times before it crosses the threshold $\gamma$ at,

$$
\tau_{\gamma} \triangleq \inf \left\{k \geq 1: W_{k} \geq \gamma\right\} .
$$

We call $\tau_{\gamma}$ the First Passage Time (FPT). The overshoot $\Gamma=W_{\tau_{\gamma}}-$ $\gamma$. Let

$$
\begin{aligned}
\tau_{0} & \triangleq \inf \left\{k: k>\tau_{\gamma} ; W_{k} \leq 0\right\}-\tau_{\gamma} \quad \text { and } \\
\eta & =\#\left\{k: W_{k} \geq \gamma ; \tau_{\gamma} \leq k \leq \tau_{\gamma}+\tau_{0}\right\} .
\end{aligned}
$$

During time $\eta$ (called a batch) a local node transmits to the fusion node. Thus, these are the times during which the fusion node will most likely declare a change. The overshoot $\Gamma$ can have significant impact on $\eta$.

It has been shown in [13] that the point process of exceedances of $\gamma$ by $W_{k}$, converges to a compound Poisson process as $\gamma \rightarrow \infty$. The points appear as clusters. The intervals between the clusters have the same distribution as that of $\tau_{\gamma}$ in $(6$ and the distribution of $\eta$ in (7) gives the distribution of the size of the cluster, i.e., the batch of the compound Poisson process. Since, one has to choose large values of $\gamma$ to keep $P_{F A}$ small, a batch Poisson process provides a good approximation in our scenario.

In the next few sections we give results on the distribution of $\tau_{\gamma}$, overshoot $\Gamma$, and the distribution of the batch $\eta$ which will be used in computing $P_{F A}$.

\subsection{First Passage Time under $\boldsymbol{P}_{\infty}$}

From the compound Poisson process approximation mentioned above,

$$
\lim _{\gamma \rightarrow \infty} \mathbf{P}_{\infty}\left\{\tau_{\gamma}>x\right\}=\exp \left(-\lambda_{\gamma} x\right), x>0
$$

where, $\lambda_{\gamma}$ a positive constant.

In [2] a formula for $\lambda_{\gamma}$ was used which is computable for Gaussian distribution only. However, by solving integral equations obtained via renewal arguments ([14]), one can obtain the mean of FPT for any distribution. Epochs when $W_{k}=0$ are renewal epochs for this process. Let $L(s)$ be the mean FPT with $W_{0}=s \geq 0$. Hence $\lambda_{\gamma}=1 / L(0)$. Then from renewal arguments:

$$
L(s)=F_{Z}(-s) L(0)+\int_{-s}^{\gamma} L(s+z) d F_{Z}(z) d z+P[Z>\gamma-s] .
$$

The equation is obtained by conditioning on $Z_{0}=z$. If $Z_{0} \leq-s$, then $W_{1}=0$, providing the first term on the right. If $Z>\gamma-s$, then the threshold is approached in one step only, providing the last term. This equation can be solved recursively on $L(s), 0 \leq s \leq \gamma$. An algorithm provided in [9] can be used to compute 9] efficiently. Table 1 provides $E\left[\tau_{\gamma}\right]$ for Pareto distribution with $K=2.1$ and Gaussian distribution with $E Z_{k}=-0.5$ and $\operatorname{var}\left(Z_{k}\right)=1$. We see that as $\gamma$ increases $E\left[\tau_{\gamma}\right]$ for Gaussian distribution becomes much larger than for the Pareto distribution. This implies that $P_{F A}$ for the Gaussian distribution should be much less than for Pareto, $K=2.1$ if $\gamma$ is large.

\begin{tabular}{|c|r|r|}
\hline$\gamma$ & $E\left[\tau_{\gamma}\right]$ Gauss & $E\left[\tau_{\gamma}\right]$ Pareto \\
\hline 5 & 930 & 800 \\
6 & 2551 & 1100 \\
7 & 6950 & 1455 \\
8 & 19020 & 1880 \\
\hline
\end{tabular}

Table 1: Mean FPT $E\left[\tau_{\gamma}\right]$ for Pareto $(K=2.1)$ and Gaussian with $E Z_{k}=-0.5$. 


\subsection{Distribution of overshoot}

Next we consider mean and distribution of the overshoot $\Gamma$. From renewal equations as in 9), we can exactly compute $E \Gamma$ for any distribution. If $R(x)=E \Gamma$ with $W_{0}=x$,

$$
\begin{aligned}
R(x) & =E\left[Z_{k}-(\gamma-x) \mid Z_{k}>(\gamma-x)\right] P_{Z}\left(Z_{k}>\gamma-x\right) \\
& +\int_{y=0}^{\gamma} R(y) f_{Z}(y-x) d y+R(0) F_{Z}(-x) .
\end{aligned}
$$

Mean overshoot $E \Gamma=R(0)$. For light tails $E \Gamma$ converges quickly to a constant value as $\gamma \rightarrow \infty$.

The distribution of the overshoot for heavy tails is reported in [1]. One can show that for light tailed distributions, overshoot is exponentially distributed. One can also obtain exact distribution using renewal integral equations as in 9 and 10 . We plot the

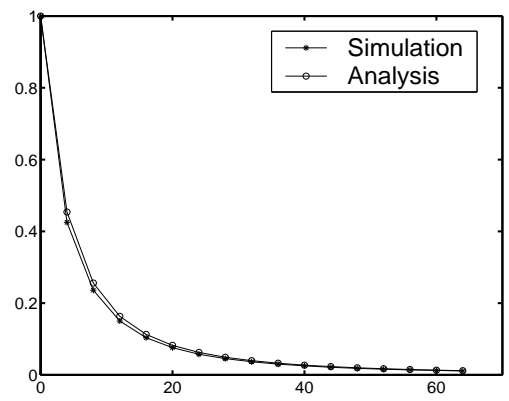

Figure 2: Complementary CDF of $\Gamma$ for Pareto $K=2.1, E Z_{k}=-0.3$ and $\operatorname{var}\left(Z_{k}\right)=1$ and $\gamma=8$

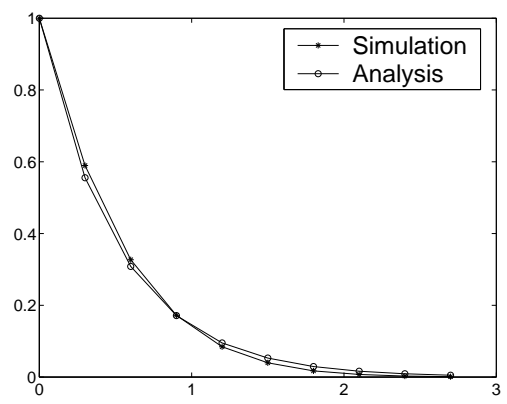

Figure 3: Complementary CDF of $\Gamma$ for $Z_{k} \sim N(-0.3,1)$ and $\gamma \geq 6$

distribution of overshoot for Pareto distribution with $K=2.1$ in Figure (2) and for Gaussian distribution in Figure (3). The mean overshoot $E[\Gamma]$ was obtained using equation $[10$. The distribution of $\Gamma$ for Pareto distribution was obtained via the result in [1] and for the Gaussian distribution by exponential distributions.

Comparing Figures (2) and (3), we see that the analytical approximations are very good. Also, the overshoot for Pareto distribution is much more than for the Gaussian distribution.

\subsection{Distribution of the Batch}

\subsubsection{Distribution of batch for heavy tail}

Theorem 2.4 of [1] provides the batch size distribution for subexponential $Z$. Figure (4) shows the plot of Batch complementary CDF for Pareto distribution with parameters $K=2.1$. One sees a good match with simulations.

\subsubsection{Distribution of batch for light tail}

Let $G_{j}(x)$ be the conditional batch distribution, $G_{j}(x)=P(\eta \leq$ $\left.j \mid W_{\tau_{\gamma}}=\gamma+x\right)$, when the overshoot is $x . G_{j}(x)$ was obtained in [2] by the Brownian Motion (BM) approximation of $\left\{W_{k}\right\}$. We use this approximation along with exponential distribution for $\Gamma$ to obtain

$$
P(\eta \leq j)=\int_{0}^{\infty} G_{j}(x) \frac{1}{E[\Gamma]} \exp \left(-\frac{x}{E[\Gamma]}\right) d x
$$

for light tailed distributions. Figure (5) plots the distribution of $\eta$ for $Z_{k}$ with Laplace distribution via (11) and via simulations.

For Lognormal distribution, which can be approximated via both heavy tailed and light tailed approximations provided above, 11 provides a better approximation.

Comparing Figures (4) and (5) one sees that the batch size for a Pareto distribution is larger than for a Laplace distribution even when they have same mean and variance. This is a direct consequence of having larger overshoots.

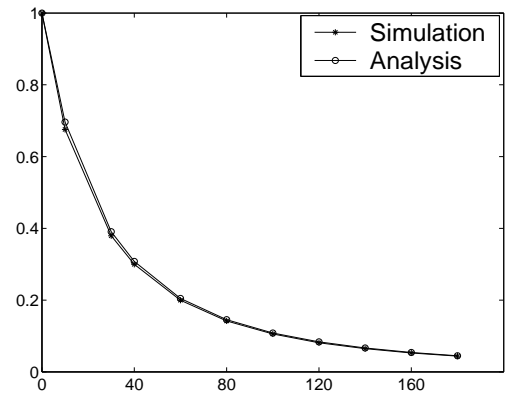

Figure 4: Complementary CDF of Batch $\eta$ for Pareto $K=2.1, E Z_{k}=-0.3$ and $\operatorname{var}\left(Z_{k}\right)=1$ and $\gamma=15$.

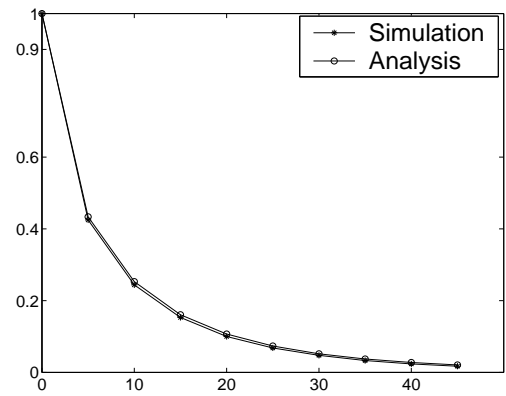

Figure 5: Complementary CDF of Batch $\eta$ for Laplace $Z_{k}$ with $E Z_{k}=-0.3$ and $\operatorname{var}\left(Z_{k}\right)=1$ and $\gamma \geq 7$.

\subsection{False Alarm Analysis}

The false alarm in DualCUSUM can happen in two ways: one within a batch (we denote its probability by $\tilde{p}$ ) and another outside it, i.e., due to $\left\{Z_{M A C, k}\right\}$. We will compute these later on. Now, we compute the $P_{F A}$ from these quantities.

From the assumptions made and the above approximation, the inter-arrival time of the batches in the system (at the fusion center) is exponentially distributed with rate $L \lambda_{\gamma}$ (because the processes $\left\{W_{k, l}\right\}$ are independent for different nodes each generating batches as Poisson processes with rate $\lambda$ ). Then, the number of batches appearing before the time of change is a Poisson random variable with parameter $L \lambda_{\gamma} i$, when $T=i$. In the following, we will show 
that the time to FA outside a batch is exponentially distributed with parameter $\lambda_{0}$ (to be defined below). Therefore, if $T \sim \operatorname{Geom}(\rho)$, then one can show that:

$$
P_{F A}=1-\frac{e^{-\left(\lambda_{0}+\lambda_{\gamma} L \tilde{p}\right)} \rho}{1-e^{-\left(\lambda_{0}+\lambda_{\gamma} L \tilde{p}\right)}(1-\rho)} .
$$

Similarly, one can obtain expression for $P_{F A}$ when $T$ is not geometric.

\section{False Alarm within a Batch:}

We have seen above that for light tailed $Z_{k}$, the $E\left[\tau_{\gamma}\right]$ is large and the batch sizes are small. Thus, the batches by different local nodes do not overlap. However, it is not true for heavy tailed distributions. Thus we compute $\tilde{p}$ for the two cases separately.

\section{Light Tailed}

The false alarm probability $(\tilde{p})$ within a batch, can be computed as, $\tilde{p} \approx \sum_{i=1}^{\infty} P(\eta=i) P(\mathrm{FA} \mid \eta=i)$, where $P(\mathrm{FA} \mid \eta=i)$ represents the probability of FA (CUSUM at the fusion center crossing $\beta$ ) in $i$ transmissions when one local node is already transmitting, i.e., $Y_{k}=b+Z_{M A C, k}$. If $\tau_{\beta}$ is the FPT variable at the fusion center, then, $P(\mathrm{FA} \mid \eta=i)=P\left(\tau_{\beta} \leq i\right)$. Since $\eta$ is small for negative drift under $f_{0}$ ( $D$ in (4) is chosen that way) we use integral equations to compute the distribution of $\tau_{\beta}$ for observations $Y_{k}$ given in this paragraph.

Table 2 gives the comparison of the $P_{F A}$ values obtained via (12) and simulations for light tailed distributions Gaussian and Laplace. It turns out that the expression is also valid for heavy tails like Lognormal (also shown in Table2). One can see a good match.

\section{Heavy Tailed}

Now, we use different arguments to compute $\tilde{p}$ and then use it in [12. For simplicity, in the following, the fusion center is assumed to use (3) for detection and not nonparametric CUSUM. From [2], the optimal choice of $I$ is found to be always greater than 1 .

Let $m$ be the minimum number of sensors required to make drift of $F_{k}$ positive. We denote the drift, with $m$ nodes transmitting, by $\mu_{m}$. Then we approximate $\tilde{p}$ by the probability that $F_{k}$ will have positive drift during a batch and that the batch lasts for $\beta / \mu_{m}$ time (the time needed for $F_{k}$ to cross $\beta$ when the drift is $\mu_{m}$ ) after $m$ sensors start transmitting. We use this approximation to compute $P_{F A}$ for Pareto $K=2.1$ distribution. This is also provided in Table 2 We see that the approximation is indeed good for Pareto $K=2.1$.

\begin{tabular}{|r|r|r|r|r|r|r|}
\hline & $L$ & $I$ & $\gamma$ & $\beta$ & $\begin{array}{r}P_{F A} \\
\text { Anal. } \\
\times 10^{-4}\end{array}$ & $\begin{array}{r}P_{F A} \\
\text { Sim. } \\
\times 10^{-4}\end{array}$ \\
\hline Gauss & 5 & 2 & 15 & 18 & 1.22 & 1.1 \\
& 10 & 2 & 15 & 18 & 2.43 & 2.28 \\
\hline Laplace & 6 & 2 & 16 & 16 & 2.57 & 2.06 \\
& 12 & 3 & 16 & 16 & 0.66 & 0.55 \\
\hline Log- & 5 & 2 & 25 & 20 & 1.47 & 1.76 \\
normal & 10 & 2 & 25 & 20 & 2.97 & 3.5 \\
\hline Pareto & 5 & 3 & 30 & 30 & 1.93 & 1.77 \\
K=2.1 & 5 & 3 & 50 & 50 & 0.23 & 0.25 \\
\hline
\end{tabular}

Table 2: $P_{F A}$ for various distributions using 4 at the local node and 3 at the fusion node: $E Z_{k}=-0.3, \operatorname{var}\left(Z_{k}\right)=1, \rho=0.005$ and $b=1$.

\section{False Alarm outside a Batch}

In the absence of any transmission from the sensors, $Y_{k} \sim N\left(0, \sigma_{M A C}^{2}\right)$ if $Z_{M A C} \sim N\left(0, \sigma_{M A C}^{2}\right)$, where $N\left(0, \sigma_{M A C}^{2}\right)$ denotes Gaussian distribution with mean 0 and variance $\sigma_{M A C}^{2}$. Hence, $F_{k}$ has negative drift. Thus the time to first reach $\beta$, i.e., time till FA, is approximately exponentially distributed with parameter $\lambda_{0}$ which can be obtained from Section 3.2

\subsection{Comparative overall performance}

The effect of tail of $Z_{k}$ on FPT, overshoot and batch size was shown in the previous sections. This causes much larger $P_{F A}$ for heavy tailed $Z_{k}$ compared to the light tailed distributions for same mean and variance. This gets reflected into large $E_{D D}$ for heavy tailed distributions for a given $P_{F A}$. Table 3 confirms these conclusions as the $E_{D D}$ for a light tailed system is much smaller as compared to the one from a heavy tailed system. The individual systems are optimized to make sure that each performs at its best.

Table 4 shows the comparative performance of paramentric and nonparametric DualCUSUM's for given $f_{0}$ and $f_{1}$. The difference in performance is most pronounced when the tail of $f_{0}$ is heaviest, i.e., for $K=7$, while the performance is same for Gaussian distributions on which log likelihood function has no effect.

Note that in Table 4 the variance of $Z_{k}$ is different for parametric and nonparametric CUSUMs. The overall effect is thus a combination of the effect of tails and that of the variances. However, as can be seen from the table, the effect of tail dominates and the general conclusion that light tailed systems are better, still holds.

$E_{D D}$ in Tables 3 and 4 are computed via simulations. However in the next section we theoretically evaluate $E_{D D}$ and then compare with the simulated values.

\begin{tabular}{|r|r|r|r|}
\hline$\rho$ & $P_{F A}$ & $\begin{array}{r}E_{D D}^{*} \\
\text { Gauss }\end{array}$ & $\begin{array}{r}E_{D D}^{*} \\
\text { Pareto } \\
\mathrm{K}=2.1\end{array}$ \\
& & & \\
\hline $5 \mathrm{e}-4$ & $\mathrm{e}-2$ & 29 & 36 \\
$5 \mathrm{e}-4$ & $\mathrm{e}-3$ & 33 & 49 \\
$1 \mathrm{e}-4$ & $\mathrm{e}-3$ & 41 & 95 \\
\hline
\end{tabular}

Table 3: Comparison of $E_{D D}^{*}$ of Gaussian $\left(I^{*}=3\right)$ and Pareto $\left(I^{*}=4\right)$ with $L=5$, $\mathscr{E}_{0}=5, E Z_{k}=-0.5, \operatorname{var}\left(Z_{k}\right)=1$ and $Z_{M A C}=N(0,1)$.

\subsection{Computation of $E_{D D}$}

The detection delay, $E_{D D}$, at the fusion node, after the change has occurred, can be written as,

$$
E_{D D}=E\left[(\tau-T)^{+}\right]=E[\tau-T \mid \tau \geq T]\left(1-P_{F A}\right) .
$$

The $E_{D D}$ is atleast equal to the time it takes for some of the sensors to transmit and make the drift of $F_{k}$ positive. After that the additional delay is due to the time it takes for $F_{k}$ to cross $\beta$ with this positive drift, or due to the additional positive drift given by transmissions due to the other remaining sensors. This concept is used to compute the $E_{D D}$ in the following.

When $\mu=E Z_{k}>0$, the time $\tau_{\gamma}$ for $W_{k}$ at a local node to cross threshold $\gamma$ satisfies, $E\left[\tau_{\gamma}\right] / \gamma \rightarrow 1 / \mu$ as $\gamma \rightarrow \infty$. Thus for large $\gamma$, $\tau_{\gamma} \sim \gamma / \mu$. Again for large $\gamma$, one can invoke Central Limit Theorem and approximate $\tau_{\gamma}$ by a Gaussian random variable: $\tau_{\gamma} \sim$ $N\left(\frac{\gamma}{\mu}, \frac{\sigma^{2} \gamma}{\mu^{3}}\right)$, where, $\sigma^{2}=\operatorname{var}\left(Z_{k}\right)$.

Let $\mu_{l}$ be the drift of fusion CUSUM $F_{k}$ when $l$ local nodes are transmitting.

Let $t_{i}$ be the point at which the drift of $F_{k}$ changes from $\mu_{i-1}$ to $\mu_{i}$, i.e., when the drift of $F_{k}$ is already $\mu_{i-1}$ and one additional node (out of the remaining $L-i+1$ ) starts transmitting. Call $t_{i}$ the ith transition epoch. Let $\tau_{x, 1}, \tau_{x, 2}, \ldots, \tau_{x, L}$ be iid with distribution of $\tau_{x}$. Then $t_{1}$, the time at which the first transmission happens, can be written as, $t_{1}=M(L, \gamma)=\min \left(\tau_{\gamma, 1}, \tau_{\gamma, 2}, \ldots, \tau_{\gamma, L}\right)$. Let $m(L, \gamma)=$ $E[M(L, \gamma)]=E\left[t_{1}\right]$. Then $E\left[t_{2}\right] \approx m(L, \gamma)+m(L-1, \gamma-m(L, \gamma) \mu)$. The term $m(L-1, \gamma-m(L, \gamma) \mu)$ represents the mean time for one 
of the remaining $L-1$ sensors to cross $\gamma$ given that their respective CUSUMs have run for $t_{1}$ slots, i.e., if $\gamma_{L-1}=\gamma-m(L, \gamma) \mu$, then $m(L-1, \gamma-m(L, \gamma) \mu)=E\left[\min \left(\tau_{\gamma_{L-1}, 1}, \tau_{\gamma_{L-1}, 2}, \ldots, \tau_{\gamma_{L-1}, L-1}\right)\right]$. In $t_{1}$ slots each of the $W_{k}$ has moved up by approximately $E\left[t_{1}\right] \mu$ and hence the correction. This way we can compute the approximation to mean of each of the transition epochs:

$$
E\left[t_{i}\right]=\sum_{l=L}^{i} m\left(l, \gamma_{l}\right),
$$

where, $\gamma_{L}=\gamma$ and $\gamma_{l}=\gamma_{l+1}-\mu . m\left(l+1, \gamma_{l+1}\right)$ and $i \leq L$. It may happen for large values of $\mu$ that, $m\left(k, \gamma_{k}\right) \leq 1$; for some $k$. In that case we set $m\left(i, \gamma_{i}\right)=0 ; k \geq i \geq 2$.

\begin{tabular}{|r|r|r|r|}
\hline$f_{0} \rightarrow f_{1}$ & $L / I$ & $\begin{array}{r}E_{D D}^{*} \\
\text { nonparametric }\end{array}$ & $\begin{array}{r}E_{D D}^{*} \\
\text { parametric }\end{array}$ \\
\hline $\begin{array}{r}\text { Pareto } x_{m}=1 \\
K=7 \text { to } K=3\end{array}$ & $5 / 4$ & 54.8 & 4.4 \\
\hline $\begin{array}{r}\text { Pareto } x_{m}=1 \\
K=40 \text { to } K=30\end{array}$ & $5 / 4$ & 69.1 & 24.9 \\
\hline Gaussian $\sigma=1$ & $5 / 3$ & 10.1 & 10.1 \\
$E Z_{k}=0$ to $E Z_{k}=0.6$ & & & \\
\hline
\end{tabular}

Table 4: Comparative performance of parametric and nonparametric DualCUSUM for $P_{F A}=0.01$ with $\rho=0.05, \mathscr{E}_{0}=7.61$, and $Z_{M A C}=N(0,1)$.

Now define, $\bar{F}_{i}=E\left[F_{t_{i}-1}\right]$, the mean value of $F_{k}$ just before the transition epoch $t_{i}$. Then a recursion can be written to approximately compute the $\bar{F}_{i}$ :

$$
\bar{F}_{k}=\bar{F}_{k-1}+1_{\left\{\mu_{k-1}>0\right\}}\left[\mu_{k-1} m\left(L-k+1, \gamma_{L-k+1}\right)\right] ; \quad \bar{F}_{1}=0 .
$$

Let, $j=\min \left\{i: \mu_{i}>0\right.$ and $\left.\frac{\beta-\bar{F}_{i}}{\mu_{i}}<E\left[t_{i+1}\right]\right\}$, then $E_{D D}$ can be approximated by,

$$
E_{D D} \approx E\left[t_{j}\right]+\frac{\beta-\bar{F}_{j}}{\mu_{j}}
$$

where, $m(k, x)$ can be computed as, $m(k, x)=\sum_{i=0}^{\infty} P\left(\tau_{x}>i\right)^{k}$.

Since, the strong law of large number and the central limit theorem suffice to build the approximations, the $E_{D D}$ is independent of the distribution of $Z_{k}$ but depends only on its mean and variance. The results are shown in Table 5 for different distributions. The second column is our approximation (14) and the rest are obtained via actual system simulations. It can be seen that, as $\gamma$ reaches 50 , the $E_{D D}$ of all the distributions considered is nearly 147 .

\begin{tabular}{|r|r|r|r|r|r|}
\hline$\gamma$ & $\begin{array}{r}E_{D D} \\
\text { Anal. }\end{array}$ & $\begin{array}{r}E_{D D} \\
\text { Gauss }\end{array}$ & $\begin{array}{r}E_{D D} \\
\text { Laplace }\end{array}$ & $\begin{array}{r}E_{D D} \\
\text { Log }-\end{array}$ & $\begin{array}{r}E_{D D} \\
\text { nareto } \\
K=3\end{array}$ \\
\hline 5 & 5.3 & 9.1 & 9.3 & 9.3 & 10.7 \\
8 & 11.4 & 16.6 & 16.8 & 16.9 & 18.7 \\
15 & 30.3 & 36.3 & 36.5 & 36.7 & 38.5 \\
50 & 146.7 & 146.8 & 147.1 & 147.6 & 150.5 \\
\hline
\end{tabular}

Table 5: Comparison of $E_{D D}$ for various distributions: $L=10, I=1, \beta=\gamma$ $E Z_{k}=-0.3, \operatorname{Var}\left(Z_{k}\right)=\mathbf{1}$ and $b=1$.

\section{CONCLUSIONS}

We have proposed an energy efficient distributed change detection scheme in [2] which uses the physical layer fusion technique and CUSUM at the sensors as well as at the fusion center. In this paper we extend the algorithm to also include the nonparametric CUSUM. We have theoretically computed the probability of false alarm and mean delay in change detection. The analytical results provide a good approximation which can then be used to choose the optimal parameters using the optimization technique in [2].

\section{REFERENCES}

[1] S. Asmussen, "Subexponential asymptotics for stochastic processes: extremal behavior, stationary distribution and first passage probabilities", Ann. Appl. Prob. Vo. 8, No. 2, pp. 354-374, 1998.

[2] T. Banerjee, V. Kavitha and V. Sharma, "Energy efficient change detection over a MAC using physical layer fusion", In Proc. of IEEE ICASSP, Las Vegas, Apr. 2008.

[3] O. J. Boxma and J. W. Cohen, "The Single Server Queue: Heavy Tails and Heavy Traffic In self-similar Network Traffic and Performance Evaluation, K. Park and W. Willinger (ed.), Wiley, N.Y., 2000.

[4] B. E. Brodsky and B. S. Darkhovsky, "Nonparametric methods in change point problems", Kluwer Acad. Pub.s, Springer, 1993.

[5] J.-F. Chamberland and V.V. Veeravalli, "Detection in Sensor Networks", IEEE Sig. Proc. Magazine, May 2007.

[6] T. L. Lai, "Sequential Change Point Detection in Quality Control and Dynamical Systems", Journal of the Royal Statistical Society, Series B (Methodological), Vol. 57, No. 4, pp. 613-658, 1995.

[7] K. B. Lataief and W. Zhang, "Cooperative Spectrum Sensing in Cognitive Wireless Communication Networks, (ed.) E. Hossain and V. K. Bhargava, Springer, 2007.

[8] G. Lorden, "Procedures for reacting to a change in distribution", Ann. Math. Statist. Vol. 41, pp. 1897-1908, 1971.

[9] A. Luceno and J. Puig-Pey, "Evaluation of the run-length probability distribution for CUSUM charts: assessing chart performance", Technometrics, Vol. 42, No. 4, pp. 411-416, 2000.

[10] Y. Mei, "Information bounds and quickest change detection in decentralized decision systems", IEEE Trans. on I. T., Vol. 51, pp. 2669-2681, Jul. 2005 IEEE Trans. on Sig. Proc., Vol. 54, No. 2, Feb. 2006.

[11] G. V. Moustakides, "Optimal stopping times for detecting changes in distribution", Ann. Statist. 14 1379-1387, 1986.

[12] E. S. Page, "Continuous inspection schemes", Biometrica, Vol. 41, No. 1/2, pp. 100-115, Jun. 1954.

[13] H. Rootzen, "Maxima and exceedances of stationary Markov chains", Adv. in Appl. Prob., Vol. 20, No. 2, pp. 371-390, Jun. 1998.

[14] S. Ross, "Stochastic Processes",2nd Ed., Wiley, 1996.

[15] V. Sharma and A. K. Jaya Prakasam, "An efficient algorithm for cooperative spectrum sensing in cognitive radio networks", in Proc. National Conf. on Comm. (NCC) Jan. 2009, Guwahati, India.

[16] A. N. Shiryaev, "On optimal methods in quickest detection problems”, Theory Probab. Appl., Vol. 8, pp. 22-46, 1963.

[17] R. Tantra and A. Sahai, "SNR walls for signal detection", IEEE Jour. on Selected Topics in Signal Proc., Vol. 2, pp. 4-17, Feb. 2008.

[18] A. Tartakovsky and V. V. Veeravalli, "Quickest change detection in distributed sensor systems", Proc. of the 6th Int. Sym. on Inf. Fusion, Australia, Jul. 2003,. 756-763

[19] V. V. Veeravalli. "Decentralized quickest change detection", IEEE Trans. on IT, 47(4): 1657-65, May 2001.

[20] L. Zacharias and R. Sundaresan, "Decentralized sequential change detection using physical layer fusion", Proc. ISIT, France, Jun. 2007. 